\title{
Isotope effect on the dynamics of hydrophilic solutions at supercooled temperatures
}

\author{
Jorge H. Melillo ${ }^{1}$, Silvina Cerveny ${ }^{1,2^{*}}$ \\ ${ }^{1}$ Centro de Física de Materiales (CSIC-UPV/EHU)-Material Physics Centre (MPC), Paseo Manuel de \\ Lardizábal 5 (20018), San Sebastián, Spain. \\ ${ }^{2}$ Donostia International Physics Center (DIPC), Paseo Manuel de Lardizabal 4 (20018), San Sebastián, Spain. \\ *email: silvina.cerveny@ehu.es
}

Numerous works have been done in the last years concerning the dynamics of water in hydrophilic solutions at supercooled temperatures using broadband dielectric spectroscopy ${ }^{1,2}$. However, there are some effects on water dynamics that were not explored in detail. In particular, in this talk, we will analyze the isotope effects on the dynamics of water in these solutions. The solutes used were tri-propylene glycol (3PG), penta-ethylene glycol (5EG) and 1-lysine in solution with three isotopes of water $\left(\mathrm{H}_{2} \mathrm{O}, \mathrm{D}_{2} \mathrm{O}\right.$ and $\left.\mathrm{H}_{2}{ }^{18} \mathrm{O}\right)$. Using these three isotopes, we can access the effects of mass and moment of inertia as well as the impact of nuclear quantum effects (NQEs) on the dynamics of water in hydrophilic solutions. We found that nuclear quantum effects are more relevant than mass effects at both high water contents and low temperatures.

1. Swenson, J.; Cerveny, S., Dynamics of Deeply Supercooled Interfacial Water. J. Phys.: Condens. Matter 2015, 27, 033102.

2. Cerveny, S.; Mallamace, F.; Swenson, J.; Vogel, M.; Xu, L., Confined Water as Model of Supercooled Water. Chemical Reviews 2016, 116 (13), 7608-7625. 